\title{
Erratum to: An analytical solution for the swing-by problem
}

\author{
G. B. Valsecchi' ${ }^{1,2}$ - E. M. Alessi' ${ }^{2}$ A. Rossi ${ }^{2}$
}

Published online: 8 September 2015

(C) Springer Science+Business Media Dordrecht 2015

\section{Erratum to: Celest Mech Dyn Astr (2015) 123(2) DOI 10.1007/s10569-015-9631-6}

The expression for $\cos i$ at the beginning of Section 4 is incorrect; the correct formula is:

$$
\cos i=\frac{1+U \cos \theta}{\sqrt{1+2 U \cos \theta+U^{2}\left(1-\sin ^{2} \theta \sin ^{2} \phi\right)}} .
$$

At the end of the same section, after the formula for $\Omega$, add the following sentence: Note that in this case $\lambda_{p}$ is the longitude of the planet at time $t_{b}$.

The online version of the original article can be found under doi:10.1007/s10569-015-9631-6.

$凶$ G. B. Valsecchi giovanni@iaps.inaf.it

E. M. Alessi

em.alessi@ifac.cnr.it

A. Rossi

a.rossi@ifac.cnr.it

1 IAPS-INAF, via Fosso del Cavaliere 100, 00133 Roma, Italy

2 IFAC-CNR, via Madonna del Piano 10, 50019 Sesto Fiorentino, Italy 